\title{
The effect of aging temperature on mechanical properties of banana pseudostem fiber reinforced polymer composite
}

\begin{abstract}
Polymer composites using natural fibers as the reinforcing agents have found their use in many applications. The initial development of polymer composite based on high density polyethylene (HDPE) with banana pseudostem fiber was studied based on the effect of nonaging and aging treatments. Tensile, flexural and impact tests were performed to investigate the mechanical properties of banana pseudostem fiber reinforced composite. Flexural and impact test results show that the specimen strength improved after aging treatment was applied. However, the tensile test results gave a reverse effect on the strength of the composite after aging. The specimen became more brittle when the composite was exposed to the aging temperature.
\end{abstract}

Keyword: High density polyethylene; Banana pseudostem fiber reinforced polymer composite; Mechanical properties 